\title{
BMJ Open Strategies to enhance recruitment and consent to intensive care studies: a qualitative study with researchers and patient-public involvement contributors
}

\author{
Katie Paddock (D) , ${ }^{1,2}$ Kerry Woolfall, ${ }^{2}$ Lucy Frith (D) , ${ }^{3}$ Megan Watkins, ${ }^{2}$ \\ Carrol Gamble, ${ }^{4}$ Ingeborg Welters (D) , ${ }^{5,6}$ Bridget Young ${ }^{2}$
}

To cite: Paddock K, Woolfall K, Frith L, et al. Strategies to enhance recruitment and consent to intensive care studies: a qualitative study with researchers and patient-public involvement contributors. BMJ Open 2021;11:e048193. doi:10.1136/ bmjopen-2020-048193

- Prepublication history for this paper is available online. To view these files, please visit the journal online (http://dx.doi. org/10.1136/bmjopen-2020 048193).

Received 18 December 2020 Accepted 27 August 2021

Check for updates

(C) Author(s) (or their employer(s)) 2021. Re-use permitted under CC BY-NC. No commercial re-use. See rights and permissions. Published by BMJ.

For numbered affiliations see end of article.

Correspondence to

Dr Katie Paddock;

k.paddock@mmu.ac.uk

\section{ABSTRACT}

Objective Clinical trials and studies in intensive care units (ICUs) have complex consent processes and often encounter problems in recruiting patients. By interviewing research team members about the challenges in critical care research, we aimed to identify strategies to enhance recruitment and consent to ICU studies.

Methods Semistructured interviews with UK-based researchers $(\mathrm{N}=17)$ and patient-public involvement $(\mathrm{PPI})$ contributors $(\mathrm{N}=8)$ with experience of ICU studies. Analysis of transcripts of audio-recorded interviews drew on thematic approaches.

Results Seven themes were identified. Participants emphasised the need for substitute decision-making processes in critical care studies, yet some researchers reported that research ethics committees (RECs) were reluctant to approve such processes. Researchers spoke about the potential benefits of research without prior consent (RWPC) for studies with narrow recruitment windows but believed RECs would not approve them. Participants indicated that the activity of PPI contributors was limited in critical care studies, though researchers who had involved PPI contributors more extensively were clear that their input when designing consent processes was important. Researchers and PPI contributors pointed to resource and staffing limitations as barriers to patient recruitment. Researchers varied in whether and how they used professional consultees as substitute decisionmakers, in whether they approached families by telephone to discuss research and in whether they disclosed details of research participation to bereaved relatives.

Conclusion Critical care research could benefit from RECs having expertise in consent processes that are suited to this setting, better staffing at research sites, more extensive PPI and an evidence base on stakeholder perspectives on critical care research processes. Guidance on professional consultee processes, telephoning relatives to discuss research, RWPC and disclosure of research participation to bereaved relatives could help to harmonise practice in these areas and enhance recruitment and consent to critical care studies.

\section{INTRODUCTION}

Clinical research in intensive care units (ICUs) is essential for improving care and
Strengths and limitations of this study

- The study includes the views of UK-based researchers and patient-public involvement (PPI) contributors to explore the problems that research teams encounter in recruiting and consenting to intensive care unit (ICU) studies.

- A sample of just eight PPI contributors limits the scope of perspectives included in this study, though does reflects researchers' reliance on a small pool of PPI contributors for their studies.

- Most of the studies discussed at interview were interventional and there may be distinct issues in recruiting and consenting to observational ICU studies that our findings do not cover.

- We focused on UK-based participants, so transferability of findings to other contexts may be limited.

treatments for critically ill patients. However, patients often lack decisional capacity at the point when they are recruited to critical care studies and the 'gold standard model' of consent, where an autonomous patient makes an informed decision about whether to take part in research, is not applicable. ${ }^{12}$ To make sure patients can benefit from the improvements to care that research brings, regulations have been developed in many countries to permit enrolment of incapacitated patients. ${ }^{3-5}$ These aim to balance the rights of these patients with the need for research in ICUs and other settings where patients do not have capacity. ${ }^{6}$ Box 1 below summarises the regulations governing recruitment and consent of incapacitated patients to research in England and Wales, as outlined by the Health Research Authority (HRA), while variants of these requirements apply in Scotland and Northern Ireland.

Despite regulations permitting recruitment of incapacitated patients to studies, successfully implementing research and recruiting 
Box 1 Overview of approaches to recruitment and consent seeking with incapacitated patients in England and Wales* by study type

(1) Clinical trials of investigational medicinal products (CTIMPs)

\section{CTIMPs in non-emergency situations}

Investigators can seek prospective consent from an incapacitated patient's legally designated personal representatives. Personal representatives are personally known to the patient, such as a family member or a close friend. However, if there is no representative, they are not available or they are unwilling to act (ie, you can not contact them or they do not want to make that decision), a doctor who is independent of the study can act as a legally designated professional representative and approve a patient's recruitment to a trial in certain circumstances. Researchers will usually seek consent (eg, for continued participation and further disclosure of confidential information) from the patient, if and when they regain capacity.

\section{CTIMPs in emergency situations}

When investigating treatments that must be administered urgently and it is not reasonably practicable to obtain consent from a legally designated representative, patients can be recruited into a trial without prior consent. This is known as research without prior consent. As patients recruited under this process may regain capacity to give consent, researchers are required to plan how they will involve patients in the on-going consent process. Trial participation and any relevant consent required (eg, consent for continued participation and disclosure of confidential information) should be discussed with legally designated representative, or patient if they regain capacity, as soon as possible after the patient's recruitment to the trial.

\section{(2) Other study types (non-CTIMPs)}

Other study types are those that involve the processing of personal data, administration of interviews or observations and clinical trials that are not CTIMPs.

\section{Other types of study in non-emergency situations}

Before a patient is recruited to such a study, investigators are required to seek advice from the patient's personal consultee, usually a family member, about the patient's likely wishes. If investigators are unable to identify a personal consultee, they can consult with a nominated consultee, which is usually a doctor responsible for the patient's care who has no connection to the research. When a patient recruited under a consultee process subsequently regains capacity, study participation should be discussed.

\section{Other types of study in emergency situations}

Patients can be recruited without prior advice from a consultee, provided it is not reasonably practicable to seek such advice in advance. Investigators need to seek agreement of a registered medical practitioner who is not involved in the organisation or conduct of the study - unless there is insufficient time to obtain that agreement. The consultee's advice should be sought on the participant's likely views and feelings about the study as soon as possible after recruitment. If objections are raised, the patient must be withdrawn unless doing so would pose a risk to the participant's health. When a patient recruited under a consultee process subsequently regains capacity, study participation should be discussed.

*Adapted from the UK Health Research Authority website with reference to relevant legislation. ${ }^{39-41}$ patients in critical care settings can be challenging. A review by Schandelmaier et $a l^{l}$ found that trials in emergency care and ICUs are four times more likely to be prematurely discontinued due to slow patient recruitment compared with trials outside these settings. Approximately $44 \%$ of randomised control trials do not meet their recruitment targets. ${ }^{8}$ In addition to the recruitment barriers that are widely reported in many specialties, ${ }^{9-13}$ critical care trials face numerous specific challenges. ${ }^{7} 14$ Several of these challenges arise from the time-sensitive nature of treatments in this setting, with necessarily short time windows for recruitment of patients for many studies. Recruitment often takes place out of hours, when staffing and other resourcing may not be adequate to support research demands and family members are often not available or able to act as substitute decision-makers. However, Schandelmaier $e t a l^{7}$ noted that studies in their review rarely reported detailed reasons for recruitment problems.

To better understand the challenges in recruiting and consenting incapacitated patients to research in critical care settings, we conducted in-depth qualitative interviews with critical care research staff in the UK. As we were particularly interested in understanding the interface between the legal frameworks around consent processes and the practicalities of implementing recruitment to ICU studies, interviews explored what influenced researchers when designing recruitment and consent processes, as well as their experience of recruiting to studies. Our overall goal was to identify ways that recruitment and consent could be enhanced in ICU studies. To ensure studies are designed and implemented in ways that are patient centred, researchers in a growing number of countries are now expected to involve patients and the public when designing and implementing studies. ${ }^{15-21}$ Therefore, we also interviewed people who had been patient-public involvement (PPI) contributors on ICU studies in addition to interviewing critical care researchers.

\section{METHODS}

\section{Overview}

Interviews were conducted between late 2016 and mid 2017 as one workstream within the wider mixed methods perspectives study. The perspectives study sought to develop good practice guidance on recruitment and consent processes for ICU studies. Given these pragmatic aims, the study was broadly critical realist in approach.

\section{Patient and public involvement}

This study included two PPI contributors, who were not specifically involved in this workstream.

\section{Sampling and recruitment}

We sampled UK researchers who had experience of designing or conducting ICU studies by searching the National Institute for Health Research research portfolio, 
trial registries and snowball sampling via critical care researchers and networks. Researchers were eligible if they had been involved in critical care studies which had included at least one UK centre and was in set up, ongoing or had been published within the previous 2 years. We sent researchers an invitation email outlining the perspectives study and attached a participant information sheet with further details.

PPI contributors were eligible if they had been research partners, steering group members or advisors on ICU studies. We initially identified them by asking interviewed researchers to email PPI contributors who they had recently worked with. Researchers sent PPI contributors an invitation email and a participant information sheet. We identified relatively few PPI contributors via this route, at least in part because research teams often seemed to rely on the same few PPI contributors for multiple studies. Therefore, we also placed an advertisement for potential PPI participants in a newsletter that ICUsteps, a UK charity for ICU patients and families, ${ }^{22}$ circulated to their members. A total of 15 PPI contributors responded to our requests for participants and 8 were interviewed. Five did not respond to arrange an interview date and two declined over concerns they might breach the confidentiality of the study they were involved in.

KP contacted researchers and PPI contributors who replied to the invitation, further explaining the study and arranging the interviews. Sampling of participants aimed for data saturation, the point at which further interviews no longer add to the analysis. ${ }^{23}$

\section{Interviews}

The interviews were conducted by KP, a postdoctoral research psychologist with experience in qualitative methods. Interviews were semistructured to ensure key topics were explored, while also conversational to enable participants to raise previously unanticipated issues. To avoid idealised responses, interviews focused on specific studies that participants had been involved with. The interview topic guide (box 2) was initially informed by the published literature and the research team's experience in this area and then developed iteratively over the course of interviewing. As participants were located throughout the UK, interviews were conducted via telephone. Participants gave informed consent before being interviewed and PPI contributors were offered a £25 shopping voucher to acknowledge their contribution to the study.

\section{Analysis}

Transcripts of audio-recorded interviews were checked for accuracy and pseudoanonymised prior to analysis. KP led the analysis in close consultation with BY (a psychologist and qualitative methodologist); both read all transcripts multiple times. Analysis was broadly interpretive and informed by the literature on quality and rigour in qualitative research. ${ }^{24}$ We drew on thematic approaches ${ }^{25}$ but conducted the analysis at multiple levels, from line by line coding, to consideration of participants' narratives at
Box 2 Summary of topic guides for researchers and patient-public involvement (PPI) contributors

Interviews with researchers explored their accounts of:

- Ethical, regulatory, scientific and pragmatic considerations that guided decision-making about the design of recruitment and consent methods in their studies.

- Experiences of seeking ethical approval and feedback from research ethics committees.

- Experiences of implementing different recruitment and consent processes, including the timing of recruitment, consultation and consent seeking and involvement of different staff in this process.

- Perceived barriers to implementing recruitment and consent as planned.

- Suggestions for improving recruitment and consent processes.

Interviews with PPI contributors explored their accounts

of:

- Recruitment and consent processes in intensive care unit studies they had been involved with.

- Awareness of considerations that had informed recruitment and consent processes.

- Opinions of these recruitment and consent processes.

- Awareness of any problems that arose during the studies.

Suggestions for improving recruitment and consent processes.

a holistic level in order to ensure coherence and contextualisation. The developing analysis was discussed with wider members of the study team, which included individuals with expertise in qualitative methods, bioethics, critical care, and clinical trials. We used NVivo software to assist with indexing and coding the data.

To evidence our interpretations we present data excerpts, denoting researchers with an $\mathrm{R}$ before their identification code and PPI contributors with P.

\section{RESULTS}

\section{Participant and interview characteristics}

We invited 26 researchers and interviewed $17(65 \%)$; the remaining nine researchers did not respond to invitation emails. Of the 17 interviewed researchers (10 male, 7 female), 10 were consultants and one was a specialist registrar in intensive care medicine. The remaining six had backgrounds in academic research, clinical trial management or nursing in intensive care settings. Eight had 15 or more years' experience in critical care research, seven had between 5 and 14 years and two had less than 4 years critical care research experience. A total of 15 PPI contributors responded to the study team to express an interest in participating and we interviewed eight. As we recruited PPI contributors via participating researchers and an advertisement, we are unable to report how many received invitations but did not respond. Of the eight PPI contributors (six male, two female), all except two reported that they had lived experience of ICU care as a patient, relative or both.

Interviews usually lasted between $45 \mathrm{~min}$ and $60 \mathrm{~min}$. A total of 33 ICU studies were discussed (4 observational, 
29 interventional), although some PPI contributors had difficulty remembering details of the studies they were involved in.

\section{Qualitative findings}

The present findings are organised in seven themes. We turn first to participants' accounts of the design of recruitment and consent processes, before presenting their perspectives on the practical implementation of these processes in studies. We note that the terminology used by participants often differed to that used in guidance and regulations. For brevity and reflecting the terminology of participants, we use the term consultee to refer to substitute decision-makers in both clinical trials of investigational medicinal products (CTIMPs) and non-CTIMPs.

\section{Designing recruitment and consent processes}

Designing consent processes was felt to be straightforward but often lacked PPI

Unsurprisingly, researchers spoke of regulatory requirements as an important influence on the design of their studies. Some specifically mentioned following a three tier consent 'hierarchy' (R20) in which informed consent from patients was regarded as the most acceptable process, with consultation with a personal consultee as intermediate and use of professional consultees reserved for instances when patient informed consent and a personal consultee process was not possible.

The hierarchy is, er, patient consent, if patient consent is not possible then declaration by a personal consultee. If the personal consultee is either not available or it's not appropriate to consult them, it's a declaration by a nominated [professional] consultee... a critical care consultant who is not immediately, who is not involved in the research study and is not immediately involved in the care of the patient. (R20)

Beyond this hierarchy, researchers commented that the design of the recruitment and consent processes was relatively straightforward and largely determined by study type and the overall research aims, 'it's dictated by what the study is and what you're err trying to demonstrate' (R11). They carried forward processes that they had used in previous studies 'we just really used the same process for the second study, it was, you know, pretty much accepted' (R15) and emphasised the many years of ICU clinical and research experience that they and their teams had to draw on when designing recruitment and consent processes.

Several researchers described having consulted with PPI contributors about their studies. A few believed that PPI contributors had an important role in informing consent processes. For example, R19 commented that PPI contributors had influenced their research team's decision to take a 'proportionate' approach to the consent process in their study of using a professional consultee process when relatives were unavailable or speaking with them would pose an 'unnecessary or excessive burden on relatives at a difficult time'. However, most researchers indicated that consultation with PPI contributors had largely involved them commenting on study patient information materials rather than directly informing the design of recruitment and consent processes. While one researcher said that PPI input 'wasn't necessary' (R11), more commonly researchers believed PPI input to the design of recruitment and consent processes was limited because 'you needed to be a clinician to understand the parameters' (R13).

Echoing the accounts of researchers, PPI contributors mostly described having limited input to the design of recruitment and consent processes with their roles largely being to comment on patient and family information materials and attend study steering group meetings. Also echoing the accounts of some researchers, several PPI contributors commented that their input was limited by their lack of clinical knowledge. However, some also linked their limited input to how research teams implemented PPI. For example, PPI being sought after the design of studies was largely settled, studies only having one PPI member and a lack of support and training for PPI contributors, 'there's no training for PPI... it can be a very daunting environment for someone who is perhaps retired and has come from a relatively 'normal' background' (P25). One PPI contributor also commented that some critical care researchers were 'half-hearted' (P6) about PPI and did not use it to its full potential.

Anticipated challenges of gaining ethics approval influenced the design of some studies

Researchers' experiences of preparing for and gaining research ethics committee (REC) approval for their studies were mixed. Some indicated that gaining REC approval was 'very straightforward' (R11). This included several researchers who had obtained approval to use substitute decision-makers in their studies. Outlining the REC's rationale for approving enrolment to their study via a 'declaration by a nominated consultee 'where necessary, R20 explained:

The reason the ethics committee agreed... we were keen to have a blood test on the day of severe acute [name of condition] and... they also accepted that the relatives are not always available and even if they are available, they may be too distressed.

Some researchers commented that they and their study teams had become adept in understanding what ethics committees would or would not approve. One described having 'built up a relationship' (R7) with an REC and working in a mutual learning cycle, whereby the REC gained experience in reviewing studies involving incapacitated patients, while their research team used the REC's feedback over numerous previous studies to cumulatively fine tune their processes and patient materials for new applications. Similarly, another researcher spoke of selecting RECs who were experienced in dealing with ICU studies and avoiding ones without such experience: if 
you have a difficulty it's because you've gone to the wrong REC' (R18). The feedback such researchers received from RECs on their applications largely amounted to minor revisions to patient information materials.

Others described the challenges they had encountered in gaining approval for their studies. These researchers felt RECs were 'negative' (R11) and described instances of RECs refusing to approve consent processes that were commonplace in ICU studies.

Making you justify, endlessly, why, in an incapacitated patient, you can't take a form of consent. It's like an uphill battle... Despite going and justifying the rationale, they tend to just come back and say 'no, we don't want you to do that, we want you to take written consent or we want you to try without telephone consent and if you're struggling to recruit, come back to us and then we'll give you, then we might give you telephone consent. (R4)

Commenting on the narrow time windows of many ICU studies, several researchers were interested in whether research without prior consent (RWPC) would help to address such challenges. However, one researcher described having been denied REC permission for RWPC in their study, while others commented that they had anticipated problems in ethical gaining approval for RWPC $^{\mathrm{i}}$ and so had a priori ruled out RWPC when developing their funding and ethics applications. R8 explained the potential implications for investigating the efficacy of the time-sensitive study intervention in their study.

If therapies aren't started within a specific, um, time window... their potential, um, efficacy might be lost... we're currently trying to get a sedation study funded and we think that using it very early is likely to be beneficial but we're probably going to, um, compromise that because we know we won't be able to get consent quickly enough if we don't get, um, deferred consent. (R8)

Similarly, R1 attributed their team's previous decision not to seek REC approval for RWPC in a study to the difficulty of justifying the use of RWPC. R1 linked this difficulty to a lack of evidence on stakeholder perspectives on RWPC at the time the team were designing the study and noted that evidence of stakeholders' perspectives on RWPC had since been published in the context of paediatric emergency and critical care studies.

We just couldn't see how we could quite justify it [RWPC], but I think we might have had a go at justifying it by using a lot of the other work that's going on at the moment, especially through some of [name of researcher]'s stuff... I would have been a bit braver. (R1)
Without recourse to RWPC, R1 suggested that their study was 'delaying treatment essentially' and elaborated on the concerns this raised: 'my main worry.... in the real world you would be starting that protocol at the point of when they've met the eligibility criteria... rather than an hour and a half later' (R1). Speaking of the 'lack of clarity' regarding RWPC as a 'major, major challenge for critical care research', R4 pointed to differences in attitudes towards RWPC between the HRA (the national authority in the UK that oversees governance in healthcare research) and individual ethics committees.

The people at the top were very clearly saying if the [consent] process is delaying when you feel this biological plausibility of the intervention should occur, you should be going to the ethics committee and saying, I need to have emergency provision because of that, whereas my experience is when you go to individual ethics committees, that that's not the view a lot of them take. (R4)

Despite these and other challenges, few researchers commented on input from PPI contributors as being relevant on such issues when seeking REC approval, although some did comment in more general terms about PPI input being helpful in discussions with ethics committees.

Make sure that there are some PPIs to feed into... that also creates very sort of strong basis for discussions with the ethics committee... otherwise you go into these things without sort of a leg to stand on if they don't feel it's the right, but if you've been able to discuss it with the ethics committee based on feedback from patients... it makes it a lot stronger. (R1)

Again, concurring with the accounts of most researchers, the PPI contributors we interviewed commented that they had provided little or no input to the REC applications for the ICU studies that they were involved with.

\section{Implementing recruitment and consent processes}

Need for alternatives to prospective informed consent from patients

In their accounts of implementing research, almost all researchers and several PPI contributors emphasised the difficulty of obtaining prospective informed consent from patients in the ICU and the need for substitute decisionmakers. Recruiting patients was often a '24/7' (R4) activity and patient eligibility for a study could change rapidly, yet staff support for research was costly and usually only available during office hours. Moreover, as both PPI contributors and researchers commented, much critical care research was time critical and study recruitment windows often did not coincide with relatives being present to act as personal consultees.

Things can happen so fast and... well if the relative's gone home and suddenly there's been a deterioration or there's, you know, where they think hang on a minute... do we want to get them on to the study.

\footnotetext{
'Several interviewees used terms such as 'deferred consent' or 'emergency provision' to refer to RWPC.
} 
Well you could be waiting six hours for the relative to come back in. (P21)

You're having to do this time sensitive, face to face with the relative at an appropriate time... then 24/7 recruitment is really challenging because it's very difficult to staff your ICU seven days a week... into the evenings... the cost of staffing you're doing is very high. (R4)

While emphasising the need for substitute decisionmakers, some researchers worried that regulation permitting such consent was precarious and spoke of the potential for future changes that could, for example, restrict the use of substitute decision-makers and make ICU research 'even more difficult to undertake' (R13) and be 'disastrous for critical care, um, because it just, it would, it would preclude research really' (R12).

\section{Challenges of using personal consultees}

Both researchers and PPI contributors described the complexities of approaching family members to act as personal consultees when patients were incapacitated. They emphasised that family members are in a 'frantic state' (R2) when patients are critically ill with feeling of anxiety going 'through the roof' (P21) and the difficulties families would have in taking in information and making a decision about a study. In this context, PPI contributors spoke of the need for researchers to be 'very gentle' (P9) when approaching families, while researchers spoke of their strong urge not to add to the families' distress and of the tensions appropriately timing the approach to families when studies often required consultation with family members very soon after a patient's admission to ICU and 'there's a lot of anxiety from the relatives'(R11). They also described family members as sometimes being reluctant to make decisions about research on behalf of the patient and as 'much more risk averse...than they [patients] would've been for themselves' (R15). When a patient had multiple family members, difficulties could also arise in establishing who should act as a patient's personal consultee and sometimes family members could not agree about whether a patient should be recruited. Several researchers also spoke of the complexities of contacting families by telephone to discuss research. A few were reluctant to do so and one researcher indicated that their REC was reluctant to permit families being approached about research over the telephone. Such complexities led some researchers to question whether involving family members in decisions about research, in the hours after a patient's admission, was necessarily superior to the use of professional consultees or RWPC.

Are we kidding ourselves that we're doing some truly ethical process where actually, would it be best to just use [RWPC] and say you know, this has been through a whole load of checks and balances? (R2)

I feel that it's more ethic, it's more ethical to use the professional consent and to gain consent from relatives when they are in the, you know, when they are kind of feeling more comfortable and less panicky about what's happened to their relatives. (R13)

\section{Challenges of using professional consultees}

In contrast, a few researchers were uneasy about the use of professional consultees for other reasons. Indeed, one spoke of making strenuous efforts to 'avoid' this process, noting concerns about how it might be perceived 'externally'. (R3)

You can see how it could be externally perceived that the consultant who signs as the professional consultee could seem to have a conflict of interest with regards to not upsetting his colleague who is the investigator... We've always usually, by hook or by crook, been able to identify a personal consultee. (R3)

Other researchers spoke about whether to 'go to a professional legal representative vs wait for the relatives' as 'a grey area' (R4). They varied in how they managed this, with some tending to reserve professional consultees for situations where family members could not be traced within a given period.

Where we know there's a next of kin but they can't come up face to face. And in that setting we would lose the patient 'cause we generally only use a professional where there are, is no next of kin at all or we're not aware of any next of kin following a, um, detailed search. (R8)

A few researchers described more flexible processes in which they extended use of professional consultees to situations where it was considered burdensome or unfeasible to ask families to make a decision.

If it looks like the family are going to come in then we'll wait for them and talk to them. But if it's going to be not feasible, or there is some reason why it would be very last minute to talk to them, and, you know, disproportionate or unfair on them, then we have an ability to take the professional advice. (R19)

R19 added that they reserved the use of professional consultees only for studies that involved treatments already in widespread clinical use, whereas for studies involving more novel treatments the patient would not be recruited without first speaking to the family. A variant on the above process involved researchers contacting the family members in advance of a patient's recruitment to inform them of a plan to recruit the patient to a study under a professional consultee process. R19 and R15 referred to using this process, although with some unease on R15's part.

If the relative was in Australia for instance we would tell them about the trial, see if they had any objections, and then we would get professional consent... but I just felt a lot more comfortable if I could catch the relatives. (R15) 
Regardless of the different perspectives and arrangements surrounding the use of professional consultees, some researchers commented that family members were generally accepting of this process when told about it 'after the event', often readily endorsing the patient's continued participation in a study. 'If you'd obtained a physician consent... then people are often very happy to say that's great, I don't have any objections to you carrying on. But if you ask them to give the consent then they would be reluctant to make a decision'. (R18)

Those PPI contributors who spoke about professional consultee processes felt that it was acceptable if the safeguards are all in place... I'm quite comfortable with that' (P17), although several expressed some reluctance or attached caveats to their support. 'I'm just a little bit wary... you just need a couple of caveats of, in there about who it is doing it, and that they're sufficiently independent to actually take in the patient's best interests' (P6).

\section{Challenges of seeking consent from patients after they regain capacity}

For patients who regain capacity after being recruited to studies under a personal or professional consultee process, it is a requirement for researchers to seek the patient's consent. However, as researchers remarked, patients 'may appear to have capacity' (R4) but this could fluctuate rapidly and they could have an array of sometimes subtle physical, cognitive and emotional difficulties influencing their capacity. As R13 noted: 'they're not critically ill but... they're still nowhere near $100 \%$ and I'm not sure they've got real energy to think about [research]'. PPI contributors echoed these comments:

If I've just been woken up from a ventilator or sedation or I'm going into high dependency from ICU do I really want papers, documents, people coming to me and talking about the study and wanting my signature. (P24)

Therefore, judging when a patient could be approached about a study was challenging: 'the ability of the research staff, even if they're very experienced, to assess that is also very, very difficult' (R4) and often involved researchers returning to assess a patient several times.

Consenting I would say is one of the most timeconsuming things that the research nurses do. So they'll often have to go back and see people, either on the ICU or in the ward, three, four, maybe five times before people actually regain capacity and they can actually consent them. (R11)

Complexities could also arise if samples or other study procedures were required soon after a patient had started to regain consciousness: 'once a patient is awake and kind of aware then you can't really approach them for a study procedure without telling them about the study... they're just realising how sick they were... it's just all too much'. (R18)
Challenges when a patient dies

With mortality rates in ICUs of around 25\%, some patients recruited to studies will not survive their illness. Where patients recruited to studies under a professional consultee process subsequently die, bereaved family members may be unaware that their deceased relative participated in a study. ${ }^{11}$ Researchers who had encountered this issue varied in their accounts of how they managed it. One indicated that their practice was to inform bereaved relatives about the patient's participation and ask them whether they wished the patient's data to be retained in the study.

By the time you've got to the relatives the patient has already died, then you would.... tell them that before [the patient] died they were put in a study, and, er, that [the family members] just need to be aware of that... you've obtained professional consent then you would give the relatives the option to say 'do you wish the data to be used or not used? (R18)

In contrast, R4 did not discuss the research with bereaved relatives and referred to published guidance to support their team's practice.

In England, under the professional legal representative... our understanding from the guidance we had was if the patient died and we didn't have any other form of consent or non-objection we could retain the patient in the study, because under the Mental Capacity Act, the professional legal representative was a valid form of documentation to include someone in a study. (R4)

In line with the advice from their PPI group, R19 similarly described their usual practice was not to inform bereaved relatives that the patient had taken part in a study, unless there were exceptional circumstances.

Our patient and public groups think that on the whole unless there's a particular reason why you need to tell the relatives, er, why something happened, they don't really want us approaching relatives of patients who've died... We do sometimes, but on average no, it's very study specific. If $[\ldots]$ a serious adverse event has occurred then we would, because we've got a duty to disclose. (R19)

While perspectives on this issue varied, researchers agreed that further guidance was needed on whether and how to disclose study enrolment and retention of data to families, when a patient recruited under a professional consultee process subsequently dies.

PPI contributors who spoke about this issue also varied in their opinions. One concurred with $\mathrm{R} 4$ and R19, commenting that it was not 'ethical to approach people who are grieving in that way' (P23). Another acknowledged the considerable difficulties involved but was doubtful whether these justified 'withholding' information about a patient's research participation from bereaved family members 'ethically that seems even more, 
Box 3 Factors that could benefit recruitment and consent to intensive care unit (ICU) studies

- Ensuring that research ethics committees which review ICU studies have relevant expertise and understanding of recruitment and consent for studies involving incapacitated patients within narrow time windows.

- Widening the scope of patient-public involvement (PPI) in ICU studies and supporting PPI contributors to feel confident in contributing to the design of recruitment and consent processes.

- Better staffing for recruiting patients.

- Evidence on the perspectives of patients, relatives and staff regarding recruitment and consent processes in adult critical care studies.

> Guidance on:

Requirements for ethical approval of research without prior consent. Circumstances in which to use a professional consent/consultee process.

Use of telephone or videoconferencing to discuss research with relatives.

Whether bereaved relatives should be informed of the patient's research participation.

er, wrong to not be able to give people that information. So, um, so I don't know'. (P6)

\section{DISCUSSION}

Our findings add empirical evidence about some of the difficulties that underlie previously reported problems in recruiting to ICU studies. Others have commented that studies in critical and emergency settings rarely reported detailed reasons for recruitment problems ${ }^{7}$ - our study provides an in-depth account of the reasons why these studies encounter recruitment difficulties and points to several ways to address these difficulties (see box 3 ).

Reflecting the regulations governing research, participants regarded informed consent by the patient as the most acceptable form of consent but emphasised that it was impossible to seek such consent when patients lacked decisional capacity. As others have similarly found, ${ }^{26} 27$ researchers reported variation in how RECs responded to their proposed consent processes, with a few reporting that some RECs were reluctant to approve consent processes that were commonly used in ICU studies. These findings point to the continued importance of ensuring RECs that review ICU studies having relevant expertise and understanding of recruitment and consent for ICU studies. For ICU studies with very narrow recruitment time windows, researchers spoke about the potential benefits of RWPC. A few had considered RWPC for their studies, but felt that RECs would not approve this process so they did not pursue it. Researchers wanted further guidance from RECs on the use of RWPC to ensure studies appropriately balanced both ethical and scientific considerations. Box 3 summarises these and other factors that could benefit recruitment to ICU studies.
The accounts of both researchers and PPI contributors indicated that PPI activity was largely limited to the development of study information materials for patients and families. Researchers who involved PPI contributors more broadly pointed to the value of their input, particularly in helping to resolve questions about consent processes, such as the use of professional consultees and the disclosure of study enrolment to bereaved families. This echoes findings from other research contexts where PPI was found to be helpful in addressing various challenges and ensuring research was acceptable and relevant to patients. ${ }^{27-29}$ Extending PPI beyond the development of study information materials to the design, planning and implementation of studies could benefit critical care research, although PPI contributors would need support and training to help them feel confident in commenting on ICU study consent processes. We have developed an accessible animation that could help as part of such training. ${ }^{30}$

In describing the implementation of recruitment and consent processes, researchers and PPI contributors were clear that recruiting patients would benefit from improved staffing and resourcing, as others have previously reported. ${ }^{14}$ Researchers were also concerned to avoid adding to the distress of families when recruiting to studies, but family members often had to be approached about research during or soon after a patient's admission to ICU. Some researchers described deliberating over when to use personal or professional consultees in such situations; where studies involved interventions that were well understood or had particularly tight timescales, a few described using professional consultees rather than personal consultees to avoid distressing families. Others regarded the use of professional consultees as questionable and avoided it altogether. Accounts also differed on the acceptability of approaching families by telephone to discuss research. Our findings resemble those from other contexts ${ }^{31-33}$ in pointing to the uncertainties and dilemmas that some researchers experience in recruiting to studies and to how researchers can gain confidence in recruiting and consenting to studies from knowing that the perspectives of patients and families have informed study protocols. There are also indications that the use of telephone consent in the UK has increased in response to the COVID-19 pandemic, given the restrictions in hospital visiting rules and risk of infection. ${ }^{34}$ Including specific advice in study protocols on issues such as the use professional consultees and approaching families by telephone to discuss research, informed by evidence on the perspectives of patients and other stakeholders and coproduced with PPI contributors, could help to address remaining uncertainty in these areas and potentially help to avoid non-recruitment of eligible patients.

Variations were also evident when participants spoke about situations where a patient had been recruited to a study under a professional consultee process and the patient later died before the family had been informed about the study. Researchers wanted further guidance on 
whether to disclose to bereaved family members that their relative had been recruited to a study. Including specific advice on this in study protocols informed by evidence on families' perspectives and codeveloped with PPI contributors could inform management of this sensitive issue. Such an approach has been useful in addressing methodological challenges in organ donation research, ${ }^{35}$ while research evidence on stakeholders' perspectives has been pivotal in informing guidance on bereavement in the context of the critical care studies involving children. ${ }^{36-38}$ Evidence on the perspectives of patients, relatives and staff regarding consent processes in adult critical care studies has been collected as part of the wider perspectives study and could be similarly informative on this and other issues.

Our study has some limitations. First, despite trying different ways to access PPI contributors with experience of ICU studies, we interviewed fewer than anticipated, at least in part because researchers tended to rely on a small pool of PPI contributors for their studies. It is possible that the perspectives of the PPI contributors who we interviewed do not reflect the breadth of PPI in ICU studies. Nevertheless, most researchers similarly described that the scope of PPI in ICU studies was limited. Second, interviews took place between late 2016 and mid 2017 and PPI input to ICU studies may have expanded since. Third, most of the studies discussed in the interviews were interventional and there may be distinct issues in recruiting and consenting to observational ICU studies that our findings do not cover. Finally, all participants were UK based. Cultural, legal and practice differences between counties means that caution is needed in transferring the findings to other countries.

In conclusion, this study offers insights on the problems that research teams encounter in recruiting and consenting to ICU studies and ways these could potentially be overcome. Our findings indicate that the critical care research could benefit from further support and guidance in the areas summarised in box 3 above. Widening the scope of PPI in ICU studies and using evidence on the perspectives of patients and relatives to inform recruitment and consent to studies may be particularly important. Insights from these can help to ensure that protocols and guidance are patient centred, as well as help research teams to feel more confident in recruiting and consenting to studies.

\section{Author affiliations}

${ }^{1}$ Faculty of Health and Education, School of Childhood, Youth and Education Studies, Manchester Metropolitan University, Manchester, UK

${ }^{2}$ Institute of Population Health, Department of Public Health, Policy and Systems, University of Liverpool, Liverpool, UK

${ }^{3}$ Faculty of Humanities and Social Sciences, Department of Law and Philosophy, University of Liverpool, Liverpool, UK

${ }^{4}$ Institute of Population Health, Department of Biostatistics, University of Liverpool, Liverpool, UK

${ }^{5}$ Institute of Life Course and Medical Science, University of Liverpool, Liverpool, UK ${ }^{6}$ Department of Critical Care, Liverpool University Hospitals NHS Foundation Trust, Liverpool, UK
Twitter Katie Paddock @KatiePaddockPhD, Kerry Woolfall @kerry_woolfall and Lucy Frith @lucy_frith

Acknowledgements The authors are grateful to the researchers and patientpublic involvement contributors who participated in the interviews. The authors are also grateful to the perspectives study advisory group.

Contributors BY, KW, LF, CG and IW designed the perspectives study. KP conducted the interviews and led the data analysis in close collaboration with BY. Author BY led on drafting the manuscript in close collaboration with KP. Authors KW, LF, CG, IW and MW contributed to further drafts of the manuscript. All authors have read and approved the manuscript.

Funding This work was supported by the UK Economic and Social Research Council (grant number ES/N006372/1).

Competing interests None declared.

Patient and public involvement Patients and/or the public were not involved in the design, or conduct, or reporting, or dissemination plans of this research.

Patient consent for publication Not required.

Ethics approval The University of Liverpool provided ethical approval (reference 0465) for this workstream of perspectives.

Provenance and peer review Not commissioned; externally peer reviewed.

Data availability statement Data will be available on a safeguarded basis via the UK Data Service repository: http://reshare.ukdataservice.ac.uk.

Open access This is an open access article distributed in accordance with the Creative Commons Attribution Non Commercial (CC BY-NC 4.0) license, which permits others to distribute, remix, adapt, build upon this work non-commercially, and license their derivative works on different terms, provided the original work is properly cited, appropriate credit is given, any changes made indicated, and the use is non-commercial. See: http://creativecommons.org/licenses/by-nc/4.0/.

\section{ORCID iDs}

Katie Paddock http://orcid.org/0000-0003-1264-5845

Lucy Frith http://orcid.org/0000-0002-8506-0699

Ingeborg Welters http://orcid.org/0000-0002-3408-8798

\section{REFERENCES}

1 Gillett GR. Intensive care unit research ethics and trials on unconscious patients. Anaesth Intensive Care 2015;43:309-12.

2 Shepherd V. Advances and challenges in conducting ethical trials involving populations lacking capacity to consent: a decade in review. Contemp Clin Trials 2020;95:106054.

3 Jackson Chornenki N, Liaw P, Bagshaw S, et al. Data initiatives supporting critical care research and quality improvement in Canada: an environmental scan and narrative review. Can J Anaesth 2020;67:475-484.

4 Furyk JS, Lawton LD, Ting JY, et al. Informed consent in emergency care research: an oxymoron? Emerg Med Australas 2017;29:110-2.

5 Luce JM, Cook DJ, Martin TR, et al. The ethical conduct of clinical research involving critically ill patients in the United States and Canada: principles and recommendations. Am J Respir Crit Care Med 2004;170:1375.

6 Dickert NW, Miller FG. Involving patients in enrolment decisions for acute myocardial infarction trials. BMJ 2015;351:h3791.

7 Schandelmaier S, von Elm E, You JJ, et al. Premature discontinuation of randomized trials in critical and emergency care: a retrospective cohort study. Crit Care Med 2016;44:130-7.

8 Walters SJ, Bonacho Dos Anjos Henriques-Cadby I, Bortolami $\mathrm{O}$, et al. Recruitment and retention of participants in randomised controlled trials: a review of trials funded and published by the United Kingdom health technology assessment programme. BMJ Open 2017;7:e015276.

9 Natale P, Saglimbene V, Ruospo M, et al. Transparency, trust and minimizing burden to increase recruitment and retention in trials: a systematic review. J Clin Epidemiol 2021;134:35-51.

10 Briel M, Olu KK, von Elm E, et al. A systematic review of discontinued trials suggested that most reasons for recruitment failure were preventable. J Clin Epidemiol 2016;80:8-15.

11 Unger JM, Vaidya R, Hershman DL, et al. Systematic review and meta-analysis of the magnitude of structural, clinical, and physician and patient barriers to cancer clinical trial participation. J Natl Cancer Inst 2019;111:245-55. 
12 van der Zande ISE, van der Graaf R, Hooft L, et al. Facilitators and barriers to pregnant women's participation in research: a systematic review. Women Birth 2018;31:350-61.

13 Hughes-Morley A, Young B, Waheed W, et al. Factors affecting recruitment into depression trials: systematic review, meta-synthesis and conceptual framework. J Affect Disord 2015;172:274-90.

14 Pattison N, Arulkumaran N, Humphreys S, et al. Exploring obstacles to critical care trials in the UK: a qualitative investigation. $J$ Intensive Care Soc 2017;18:36-46.

15 Frank L, Basch E, Selby JV, et al. The PCORI perspective on patientcentered outcomes research. JAMA 2014;312:1513-4.

16 Staniszewska S, Denegri S, Matthews R, et al. Reviewing progress in public involvement in NIHR research: developing and implementing a new vision for the future. BMJ Open 2018;8:e017124.

17 Health Research Authority. Impact of public involvement on ethical aspects of research: health research Authority/INVOLVE, 2020. Available: https://www.invo.org.uk/wp-content/uploads/2016/05/ Impact-of-public-involvement-on-the-ethical-aspects-of-researchupdated-2016.pdf

18 Canadian Institutes of Health Research. Strategy for patientoriented research: patient engagement framework: Canadian Institutes of health research, 2014. Available: https://cihr-irsc.gc.ca/ e/48413.html

19 Building a healthy Australia. Statement on consumer and community involvement in health and medical research National health and medical Research Council and the consumers health forum of Australia, 2016. Available: https://www.nhmrc.gov.au/about-us/ publications/statement-consumer-and-community-involvementhealth-and-medical-research

20 Schilling I, Behrens $\mathrm{H}$, Hugenschmidt $\mathrm{C}$, et al. Patient involvement in clinical trials: motivation and expectations differ between patients and researchers involved in a trial on urinary tract infections. Res Involv Engagem 2019;5:15.

21 Sacristán JA, Aguarón A, Avendaño-Solá C, et al. Patient involvement in clinical research: why, when, and how. Patient Prefer Adherence 2016;10:631.

22 ICUsteps. Available: https://www.icusteps.org/

23 Guest G, Bunce A, Johnson L. How many interviews are enough? an experiment with data saturation and variability. Field Method 2006;18:59-82.

24 Levitt HM, Motulsky SL, Wertz FJ, et al. Recommendations for designing and reviewing qualitative research in psychology: promoting methodological integrity. Qual Psychol 2017;4:2-22.

25 Braun V, Clarke V. Using thematic analysis in psychology. Qual Res Psychol 2006;3:77-101.

26 Dixon-Woods M, Angell EL. Research involving adults who lack capacity: how have research ethics committees interpreted the requirements? J Med Ethics 2009;35:377-81.

27 Tridente A, Holloway PAH, Hutton P, et al. Methodological challenges in European ethics approvals for a genetic epidemiology study in critically ill patients: the GenOSept experience. BMC Med Ethics 2019;20:30

28 Crocker JC, Ricci-Cabello I, Parker A, et al. Impact of patient and public involvement on enrolment and retention in clinical trials: systematic review and meta-analysis. BMJ 2018;363:k4738.

29 Forsythe LP, Carman KL, Szydlowski V, et al. Patient engagement in research: early findings from the patient-centered outcomes research Institute. Health Aff 2019;38:359-67.

30 Perspectives Study Team 2019. Research in the intensive care unit, 2020. Available: https://www.youtube.com/watch?time_continue=2\& $v=6$ HOTElv46ec\&feature $=$ emb_title

31 Donovan JL, Paramasivan S, de Salis I, Salis de I, et al. Clear obstacles and hidden challenges: understanding recruiter perspectives in six pragmatic randomised controlled trials. Trials 2014;15:5.

32 Dudley L, Gamble C, Preston J, et al. What difference does patient and public involvement make and what are its pathways to impact? qualitative study of patients and researchers from a cohort of randomised clinical trials. PLoS One 2015;10:e0128817.

33 Mann C, Chilcott S, Plumb K, et al. Reporting and appraising the context, process and impact of PPI on contributors, researchers and the trial during a randomised controlled trial - the 3D study. Res Involv Engagem 2018;4:15.

34 GenOMICC. GenOMICC study protocol version 2.4, 2020. Available: https://genomicc.org/

35 Noyes J, Mclaughlin L, Morgan K, et al. Designing a co-productive study to overcome known methodological challenges in organ donation research with bereaved family members. Health Expect 2019;22:824-35.

36 Woolfall K, Frith L, Gamble C, et al. How parents and practitioners experience research without prior consent (deferred consent) for emergency research involving children with life threatening conditions: a mixed method study. BMJ Open 2015;5:e008522.

37 Woolfall K, Frith L, Dawson A, et al. Fifteen-minute consultation: an evidence-based approach to research without prior consent (deferred consent) in neonatal and paediatric critical care trials. Arch Dis Child Educ Pract Ed 2016;101:49-53.

38 Picton A, Woolfall K, Lyttle MD, et al. Research without prior consent in paediatric emergency and critical care medicine. Paediatr Child Health 2020;30:74-8.

39 Health Research Authority. Research in emergency settings, 2020. Available: https://www.hra.nhs.uk/planning-and-improving-research/ policies-standards-legislation/research-emergency-settings/

40 Health Research Authority. Principles of consent: adults not able to consent for themselves (England and Wales), 2020. Available: http://www.hra-decisiontools.org.uk/consent/principles-ALCEnglandandWales.html

41 Health Research Authority. Mental capacity act, 2020. Available: https://www.hra.nhs.uk/planning-and-improving-research/policiesstandards-legislation/mental-capacity-act/ 